\title{
The influence of dietary protein intake on bone health and fracture risk across the lifespan: a systematic review and meta-analysis
}

\author{
A.L. Darling ${ }^{1}$, D. Wynter ${ }^{1}$, D.J. Torgerson ${ }^{2}$, C.E. Hewitt ${ }^{2}$, D.J. Millward ${ }^{1}$, S.A. Lanham-New ${ }^{1}$ \\ and R.J. Manders ${ }^{1}$ \\ ${ }^{1}$ Department of Nutritional Sciences, School of Biosciences and Medicine, Faculty of Health and Medical Sciences, \\ University of Surrey, Guildford, GU2 $7 X H$ and ${ }^{2}$ Clinical Trials Unit, University of York, York, YO10 5DD, UK.
}

The influence of dietary protein intake on health and its potential role in health and disease is still a topic of debate. To date there is still considerable controversy as to whether protein is an important nutrient for optimising skeletal integrity throughout the life-cycle. The aim of this study was to expand on a previous meta-analysis ${ }^{(1)}$ incorporating the last 10 years of research in the field of dietary protein intake and bone health in healthy humans.

PUBMED database searches were performed for research published between the $1^{\text {st }}$ January 1976 and the $22^{\text {nd }}$ January 2016 in healthy subjects. All relevant bone outcomes were included except calcium metabolism. A total of 1913 potentially relevant studies were found and of these studies 120 papers (including 53 cross sectional studies, 34 longitudinal studies and 33 supplementation trials) were identified as eligible for analysis.

Dietary protein intake explained $<1-8 \%$ of areal bone mineral content (aBMC) and areal bone mineral density (aBMD) in adults as well as $7-21 \%$ of aBMC and aBMD in children, depending on bone site (multivariate adjusted correlations used where possible). Random effects meta-analyses indicated no influence of mixed protein supplementation on adult lumbar spine (LS) aBMD (Weighted Mean Difference $(\mathrm{WMD})=0.02 ;-0.06$ to $0.09, \mathrm{I}^{2}=53 \%$ ). Meta-analyses of fracture risk studies showed no association between protein intake and relative risk $(\mathrm{RR})$ for all osteoporotic fractures combined, for mixed $\left(\mathrm{RR}=0.93 ; 0.74\right.$ to $\left.1 \cdot 17, \mathrm{I}^{2}=19 \%\right)$, animal $\left(\mathrm{RR}=0.96 ; 0.77\right.$ to $\left.1.21, \mathrm{I}^{2}=37 \%\right)$ or vegetable protein $\left(\mathrm{RR}=1.00 ; 0.88\right.$ to $\left.1.14, \mathrm{I}^{2}=3 \%\right)$.

Based on these results we can conclude that there is no association between any type of protein intake and the risk of fractures. Our analysis furthermore shows that there are small benefits of increased protein intake on bone health in adults with no indication of detrimental effects. Considering the crucial role that an adequate protein intake plays in maintaining skeletal muscle mass throughout the lifecycle and the fact that there are no indications of a detrimental effect of protein intake on the skeletal system, dietary protein can be seen as one of the most important nutrients ensuring health throughout life. Future studies however should focus more on the role dietary protein plays to optimise both skeletal integrity and skeletal muscle mass and the interplay between the two systems.

1. Darling AL, Millward DJ, Torgerson DJ et al. (2009) Am J Clin Nutr 6, 1674-1692. 